\title{
Different genetic basis for alcohol dehydrogenase activity and plasticity in a novel alcohol environment for Drosophila melanogaster
}

\author{
Sheng Pei Wang $\mathbb{1}^{1} \cdot$ David M. Althoff ${ }^{1}$
}

Received: 20 December 2019 / Revised: 15 May 2020 / Accepted: 18 May 2020 / Published online: 1 June 2020

(c) The Author(s), under exclusive licence to The Genetics Society 2020

\begin{abstract}
Phenotypic plasticity is known to enhance population persistence, facilitate adaptive evolution and initiate novel phenotypes in novel environments. How plasticity can contribute or hinder adaptation to different environments hinges on its genetic architecture. Even though plasticity in many traits is genetically controlled, whether and how plasticity's genetic architecture might change in novel environments is still unclear. Because much of gene expression can be environmentally influenced, each environment may trigger different sets of genes that influence a trait. Using a quantitative trait loci (QTL) approach, we investigated the genetic basis of plasticity in a classic functional trait, alcohol dehydrogenase (ADH) activity in D. melanogaster, across both historical and novel alcohol environments. Previous research in D. melanogaster has also demonstrated that ADH activity is plastic in response to alcohol concentration in substrates used by both adult flies and larvae. We found that across all environments tested, ADH activity was largely influenced by a single QTL encompassing the $A d h$-coding gene and its known regulatory locus, delta-1. After controlling for the allelic variation of the Adh and delta-l loci, we found additional but different minor QTLs in the 0 and $14 \%$ alcohol environments. In contrast, we discovered no major QTL for plasticity itself, including the Adh locus, regardless of the environmental gradients. This suggests that plasticity in ADH activity is likely influenced by many loci with small effects, and that the Adh locus is not environmentally sensitive to dietary alcohol.
\end{abstract}

\section{Introduction}

Phenotypic plasticity, the ability of an organism to alter its phenotype according to its environment, is argued to play important roles in many ecological and evolutionary processes, (Wang and Althoff 2019; Levis et al. 2018; Levis and Pfennig 2016), including range expansion, niche shift, population divergence, speciation, and population persistence under anthropogenic environmental changes (DeWitt and Scheiner 2004; Merila and Hendry 2014; Pigliucci 2001; Schaum and Collins 2014; Schlichting and Pigliucci 1998; West-Eberhard 2003). The first step in each of these processes depends on a population's ability to persist and

Associate Editor: Darren Obbard

Sheng Pei Wang

swang74@syr.edu

1 Department of Biology, Syracuse University, Syracuse, NY 13244, USA eventually grow under new environmental conditions. During the initial encounter, plasticity can allow a population to respond to the novel environment directly and produce better matching phenotypes before genetic changes have time to occur (Snell-Rood et al. 2010; Yeh and Price 2004). Thus, plasticity can be initially adaptive. As a population continues to locally adapt, plasticity itself can also be selected to increase, and may be instrumental in maintaining the fit between phenotype and the environment over generations (Lande 2009; Nussey et al. 2005; WestEberhard 2003).

Whether and how plasticity will evolve in novel environments relies on its underlying genetic architecture: the amount of genetic variation and the correlations with other traits under selection (Blows 2007; Gomulkiewicz et al. 2010; Kopp and Matuszewski 2014; Via et al. 1995). If plasticity is controlled by a few loci with large, additive effects, we can expect rapid evolutionary responses in plasticity and fixation at these loci (Jain and Stephan 2017). Alternatively, if plasticity is controlled by many loci with interacting effects, its evolutionary trajectory may be much slower, as well as less predictable. A complex network of 
interacting loci might constrain changes in plasticity due to the large number of simultaneous changes that need to occur. As these changes accumulate over time, there might be new plastic phenotypes that arise because the effects of a locus can depend on the allelic variation at other loci under selection, which can change as evolution proceeds (Badyaev 2005; Lande 2009; West-Eberhard 2003).

In addition, the correlation between plasticity and other traits under selection can also influence the rate and speed of plasticity evolution. For example, positive genetic correlations can accelerate the rate of adaptation if the direction of selection aligns between plasticity and other traits under selection (Chevin 2013; Schluter 1996). Genetic correlations can arise when plasticity shares causal loci with other traits under selection, or when different causal loci are physically linked on a chromosome. Thus, elucidating the genetic architecture of phenotypic plasticity is critical for understanding the role of plasticity in the evolutionary potential of populations experiencing novel environments.

Growing evidence has shown that phenotypic plasticity is genetically controlled, and can correlate extensively with other traits (Hangartner and Hoffmann 2016; McCairns and Bernatchez 2010). Two alternative genetic mechanisms have been proposed for phenotypic plasticity. In the allelic sensitivity model, the same genes control both phenotypic variation in each environment and variation in plasticity (Via et al. 1995). Alternatively, in the plasticity gene model, plasticity is an outcome of differential regulatory control of gene expression in different environments (Scheiner 1993). In reality, these mechanisms are nonexclusive and can jointly influence the plasticity of a single trait (e.g. Wei and Zhang 2017). When plasticity is mostly determined by allelic sensitivity, variation in plasticity would be highly correlated with trait values. Alternatively, plasticity based on plasticity genes would allow for separate and possibly independent evolutionary fates of trait values and their plasticity.

Although both the evolutionary importance and the genetic architecture of plasticity have received growing interests among both theoretical and empirical studies, we know much less about the evolutionary potential of plasticity in novel environments (Levis and Pfennig 2019). In order to understand past evolutionary patterns, many studies focus on historically relevant environmental gradients when studying the genetics of plasticity, and both the genetic structure of plasticity and its correlation with other traits can vary substantially, depending on the range of environments investigated (Callahan and Waller 2000; Fischer et al. 2016; Husby et al. 2011; Lacaze et al. 2009; Tonsor and Scheiner 2007). The genetic architecture of plasticity in novel environments, however, may further differ from historical environments; thus, whether we can predict the effects of plasticity in novel environments is still unclear (Chevin and Hoffmann 2017).
One way to explore the genetic architecture of plasticity is through quantitative trait mapping (QTL), especially in model systems with a sequenced genome and known gene functions. Identified QTLs provide a starting point for determining the potential causal loci with the largest effects on phenotypes. QTL regions can be further mined for genes of known functions that may provide insight into their mechanisms of action on plasticity. For example, regulatory genes may be less constrained than protein-coding genes to evolve without disruptive outcomes, and small changes in regulatory genes can have large impacts on a phenotype.

We used QTL mapping to compare and contrast the genetic basis of alcohol dehydrogenase (ADH) activity and plasticity across a range of historical and novel alcohol environments using the model organism Drosophila melanogaster. Adaptation to alcohol environments through the ADH protein has been a critical evolutionary force in many Drosophila lineages, especially in D. melanogaster, which spread around the globe by colonizing high alcohol environments associated with human activities such as agriculture (Markow 2015; Mercot et al. 1994). Some populations have even colonized winery waste with over 7\% alcohol content (Gibson et al. 1981). In addition, Wang and Althoff (2019) demonstrated that D. melanogaster can develop in substrate with even higher alcohol content (16\%) well outside the range of natural alcohol conditions experienced by larvae. Part of D. melanogaster's ability to handle alcohol is due to two well-characterized alleles at the ADH locus, fast and slow (Geer et al. 1985), that differ in the rate at which they catalyze alcohol (McDonald et al. 1980; Thompson and Kaiser 1977). Siddiq et al. (2017) and Siddiq and Thornton (2019) experimentally demonstrate this by using transgenic flies that contain one or the other allele, but also caution that these two alleles alone cannot explain all the variation in alcohol tolerance observed among populations. Thus, there are likely additional loci and other factors that are important in alcohol tolerance.

One reason for D. melanogaster's exceptional alcohol tolerance is that larvae can use the alcohol as an energy source, $90 \%$ of which is processed through the ADH pathway (Geer et al. 1985). In larvae, the ADH protein catalyzes the first two steps of the pathway: alcohol is converted to acetaldehyde and then to acetate, which is finally converted to acetyl-CoA and enters the TCA cycle (reviewed in Geer et al. 1993). Large amounts of carbon from alcohol are then converted to glycerol and fatty acids for storage (Heinstra et al. 1990), and ADH activity has been shown to control the flux from alcohol to fatty acids (Freriksen et al. 1991). Dietary alcohol can induce increased ADH activity through increased transcription (Geer et al. 1985; Mckechnie and Geer 1984), but this plasticity is not controlled by variation in the Adh-coding gene. Using mutant strains, Kapoun et al. (1990) showed that two 
regions close to the transcription-start site can regulate the transcriptional plasticity of $A d h$. However, what natural genetic variation exists for $\mathrm{ADH}$ plasticity is not well understood.

To investigate the genetic basis of $\mathrm{ADH}$ activity and plasticity, we took advantage of an established mapping resource, the Drosophila Synthetic Population Resource (DSPR). DSPR has both fine-mapping power and encompasses global genetic variation, because each mapping population was initiated by crossing eight genetically distinct founder lines from different parts of the world (King et al. 2012). Using the DSPR, we investigated the genetic basis of ADH activity and plasticity in larvae developing across different alcohol environments that includes the range found in natural conditions ( 0 and $7 \%$ alcohol) and a novel environment (14\%). We chose these concentrations because they encompass the environment in which the DSPR is raised $(0 \%)$, the most extreme concentration $(7 \%)$ for natural populations (wineries) and a value that is outside the historical range of alcohol concentrations (14\%). These three values allow us to compare and contrast the genetic basis of ADH activity and plasticity as alcohol environment changes.

\section{Materials and methods}

\section{Mapping population}

We used an established mapping population of Drosophila melanogaster, the $\mathrm{pB} 2$ subpopulation of the DSPR for this experiment (King et al. 2012). The DSPR consists of four subpopulations of partially sequenced (using restriction siteassociated DNA sequencing) recombinant inbred lines (RILs) (King et al. 2012). Each pair of the subpopulations was initiated with eight genetically diverse founders, and was maintained for 50 generations of random mating before generating the RILs, giving the mapping population finemapping power (King et al. 2012). We obtained data from 289 of the RILs from the pB2 subpopulation and five of its founder lines. All stocks were maintained on standard cornmeal-yeast-molasses medium in growth chambers at $25^{\circ} \mathrm{C}$ and $24-\mathrm{h}$ darkness.

\section{Experimental set-up}

We measured ADH (Bhatia et al. 2014) activity in larvae exposed to both historical (0 and $7 \%$ ) and novel (14\%) alcohol environments. For each RIL, the parental generation was raised and kept on food substrate with $0 \%$ alcohol. Once eclosed, the adults were allowed to mate freely among individuals with the same genotype for 3 days, so their offspring (used for the experiment) represented the inbred genotype. Mated females were transferred to fresh media for $16 \mathrm{~h}$ to lay eggs. The eggs were then manually transferred to six rearing vials, two for each treatment: 0,7 , and $14 \%$ alcohol (ethanol) in the food substrate. Each rearing vial contained 20 eggs at the maximum to keep the larval density low. If the rearing dish contained $<60$ eggs, only three rearing vials were used, one for each treatment. We setup two vials of larvae for some RILs to ensure that enough larvae survived for subsequent analyses, but only collected larvae haphazardly from one of the vials.

All rearing vials were maintained at $25^{\circ} \mathrm{C}$ and 24-h darkness, until the larvae reached the third-instar stage, and were collected for subsequent analyses. Given the slower development at higher alcohol concentrations, larvae in different treatments were collected at different times, such that they were all collected at a similar developmental stage based on size (3 days for $0 \%, 5$ days for $7 \%$, and 5.5 days for $14 \%$ ). We collected larvae by removing the top layer of the food substrate, washing them twice in clean DI water and used a paintbrush to gently roll them on a clean piece of Kimwipe to dry. Groups of five larvae (or as many as alive if $<5,3.74 \pm 1.58$ ) were collected from one rearing vial per treatment and weighed together on a microbalance. They were then flash-frozen on dry ice and stored at $-80^{\circ} \mathrm{C}$ before being assayed for ADH activity.

\section{Phenotyping}

We measured the ADH activity of larvae using spectrophotometric assays following established protocols (modified from Malherbe et al. 2005). To extract ADH from the samples, each group of larvae was homogenized and suspended in an extraction buffer $(50 \mathrm{mM}$ glycine- $\mathrm{NaOH}$, $1 \mathrm{mM}$ EDTA, pH 9.5). We scaled the amount of buffer with the number of larvae in each sample, using $50 \mu \mathrm{l}$ of extraction buffer for each larva. The suspension was then centrifuged for $5 \mathrm{~min}$ at $11,000 \mathrm{rpm}$, and only the clear supernatant was used for the spectrophotometric assay. All samples were kept on ice during preparation and stored at $-80^{\circ} \mathrm{C}$ before assaying. To measure $\mathrm{ADH}$ activity, we mixed $170 \mu \mathrm{l}$ of extraction buffer with $20 \mu \mathrm{l}$ of supernatant and $10 \mu \mathrm{l}$ of reagent buffer (extraction buffer $+5 \mathrm{mM}$ NAD $+200 \mathrm{mM}$ 2-propanol). After $30 \mathrm{~s}$ of mixing, we measured the absorbance at $340 \mathrm{~nm}$ every $3 \mathrm{~s}$ for $60 \mathrm{~s}$ using a spectrophotometer. The absorbance indicated the amount of $\mathrm{NADH}$ produced from 2-propanol and $\mathrm{NAD}^{+}$, catalyzed by the $\mathrm{ADH}$ protein, and the rate of $\mathrm{NADH}$ production corresponded linearly to ADH activity. For each assay, we used the slope coefficient from a simple linear model (absorbance $\sim$ time) to calculate the average rate of absorbance change. Each sample was measured twice as technical replicates, and we used the mean as the activity measure for that sample. We also assayed a common standard 

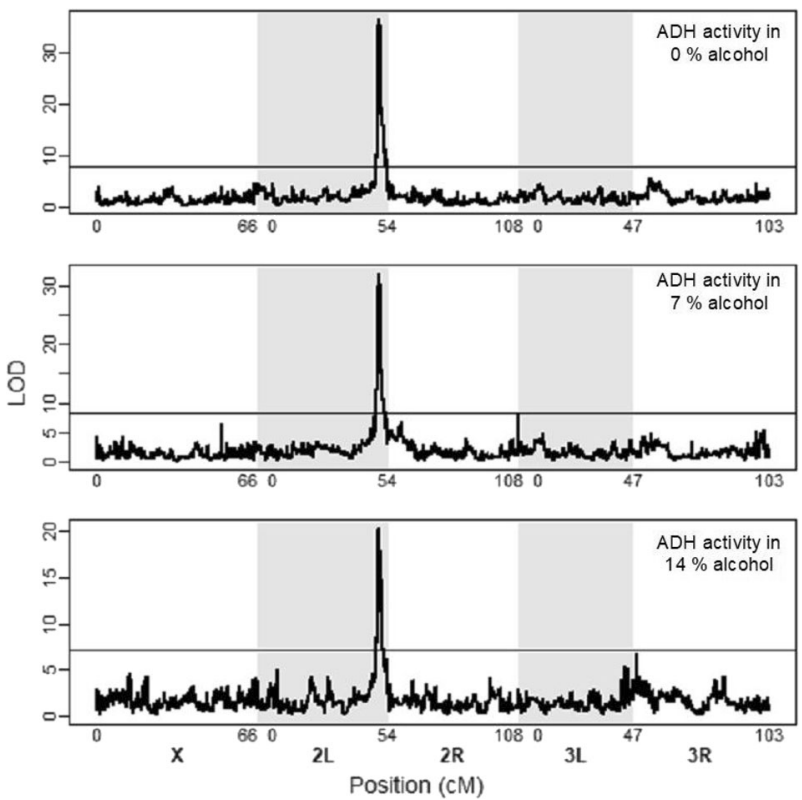

Fig. 1 QTL identified for ADH (alcohol dehydrogenase) activity in larval Drosophila melanogaster developing in substrate with 0,7 , and $\mathbf{1 4 \%}$ alcohol (top, middle, and bottom panel, respectively). Black line represents the 95\% LOD score thresholds using 1000 permutation tests. The identified QTL contains the Adh gene and its regulatory locus, delta-1.

sample prepared at the same time as the experimental samples, and this allowed us to correct for the slow deterioration of $\mathrm{ADH}$ activity and the reagent buffer over time. We further corrected all ADH activity measures by the average weight of the larvae in each sample.

\section{Plasticity measures}

We calculated three different plasticity measures for each RIL: the change in ADH activity between 0 and 7\% (p07), between 0 and 14\% (p014), and between 7 and 14\% (p714) alcohol environments. We used the discrete intervals rather than using parameters from a linear fit, because many RILs responded nonlinearly across the different intervals. Since we had no prior expectation which interval was the most biologically relevant, we used all three possible plasticity measures for subsequent QTL analyses. We also calculated the Pearson's correlation coefficients and their significance between all pairs of traits: ADH activity in each environment and the three plasticity measures. These phenotypic correlation coefficients gave us the upper limits of genetic correlations among these traits.

\section{Quantitative trait loci analysis}

We mapped QTLs for ADH activity in all three treatment environments and all three plasticity measures separately using the R packages "DSPRqtl" and "DSPRqtldataB" that were developed in concert with the DSPR (King et al. 2012; $\mathrm{R}$ Core Team 2017). We visually inspected the data for normality, and none of the traits appreciably deviated from normal distributions. Each QTL analysis produced a list of LOD scores for a set of genetic locations at regular intervals across the genome, and the LOD scores indicate how strongly variation at a genetic location associated with variation in the trait values. To determine which of the genetic locations contained significant QTLs, we performed 1000 permutation tests to determine the appropriate wholegenome LOD score thresholds, with a significance value threshold of 0.05 . We then used the conservative 2-LOD support intervals as the confidence intervals. To extract all annotated genes in each QTL region, we used the gene map table from FlyBase (FB2019_05 Thurmond et al. 2019), and we extracted the list of genes in each interval using $\mathrm{R}$ (R Core Team 2017). We further explored these genes' function and their stage-specific expression patterns through GBrowse on FlyBase (Thurmond et al. 2019).

Previous studies have shown that allelic variation in the Adh protein-coding gene and the cis-acting delta-1 region had large effects on ADH activity in flies, and existing genetic information allowed us to control for their effects in our QTL analyses (Laurie and Stam 1994). Two alleles for Adh and delta-1 were found among the founders of our population, the fast and slow alleles of $A d h$, and the high and low alleles for delta-1. The eight founders fall into three genotypic classes, fast and high, fast and low and slow and low. We assigned each RIL to one of the genotypic classes as the founder with the highest hidden Markov model probabilities at the genomic locations where Adh and delta1 resided. For any of our previous analyses that showed significant QTLs overlapping the Adh and delta-1 region, we performed additional analyses using residuals from models accounting for allelic variation in these two genes. These additional analyses allowed us to map QTLs that were not associated with Adh and the delta-1 loci with greater statistical power.

\section{Results}

For ADH activity expressed in the $0 \%$ alcohol environment, there was a single significant QTL on the left arm of the second chromosome (Table 1 and Fig. 1). This region contained the $A d h$ - coding gene and its known regulatory loci, delta-1, both of which have been established to affect ADH activity in flies (Laurie and Stam 1994). The same QTL region was also detected for ADH activity in both the $7 \%$ and the extreme 14\% alcohol environments (Fig. 1). No other significant QTL was detected for ADH activity.

Because allelic variation in both Adh and delta- 1 is well understood to affect $\mathrm{ADH}$ activity in flies, we did additional 
Table 1 Results of QTL analysis for ADH (alcohol dehydrogenase) activity and plasticity in larvae of Drosophila melanogaster developing at different alcohol concentrations in the larval-feeding substrate.

\begin{tabular}{|c|c|c|c|c|c|c|c|}
\hline $\mathrm{ADH}$ trait & Substrate alcohol conc. & Chromosome & Peak location $(\mathrm{Mb})$ & LOD & Confidence interval & Effect on phenotype & Genes \\
\hline Activity & $0 \%$ & $2 \mathrm{~L}$ & $14,800,000$ & 36.6 & $2 \mathrm{~L}: 14,380,000 . .14,960,000$ & $49.07 \%$ & Adh \\
\hline Activity & $7 \%$ & $2 \mathrm{~L}$ & $14,840,000$ & 32.2 & $2 \mathrm{~L}: 14,430,000 . .14,950,000$ & $45.59 \%$ & Adh \\
\hline Activity & $14 \%$ & $2 \mathrm{~L}$ & $14,470,000$ & 20.4 & $2 \mathrm{~L}: 14,320,000 . .14,940,000$ & $34.78 \%$ & Adh \\
\hline P07 & & \multicolumn{6}{|c|}{ No significant QTL detected } \\
\hline p014 & & \multicolumn{6}{|c|}{ No significant QTL detected } \\
\hline P714 & & \multicolumn{6}{|c|}{ No significant QTL detected } \\
\hline
\end{tabular}

Table 2 Results of QTL analysis for ADH (alcohol dehydrogenase) activity in larvae of D. melanogaster after controlling for Adh and delta-1 loci effects.

\begin{tabular}{llllllll}
\hline ADH trait & Substrate alcohol conc. & Chromosome & Peak location $(\mathrm{Mb})$ & LOD & Confidence interval & Effect on phenotype & Genes \\
\hline Activity & $0 \%$ & $2 \mathrm{~L}$ & $16,510,000$ & 8 & $2 \mathrm{~L}: 16,180,000 . .17,480,000$ & $13.93 \%$ & Unknown \\
Activity & $14 \%$ & $2 \mathrm{~L}$ & $4,590,000$ & 9.6 & $3 \mathrm{R}: 8,744,278 . .8,794,278$ & $18.41 \%$ & Unknown \\
\hline
\end{tabular}

QTL analyses after controlling for their effects. We fitted simple linear models with ADH activity as the response variable and the allelic variation as the predictor variable, and used the residuals from the models (separately for each environment) for the additional mapping analyses. This procedure allowed us to pinpoint whether the known allelic variation in Adh and delta-1 loci are the causal loci in the single QTL revealed in the previous analyses. In addition, controlling for this known allele variation gave us more power to detect additional QTLs. These analyses revealed two additional QTLs for ADH activity, one in the $0 \%$ and the other in the $14 \%$ alcohol environment (Table 2 and Fig. 2). Neither of these new QTLs overlapped with the QTL encompassing the $A d h$ gene, suggesting that allelic variation in Adh and delta- 1 was the causal variants for the mapping analyses before controlling for their effects. In the 0\% alcohol environment, the additional QTL was located on the left arm of the chromosome, $1.9 \mathrm{Mb}$ downstream from the Adh gene. This region contains a large genomic region and 143 annotated genes. Given the large number of genes covered by the region, we only explored the $100-\mathrm{kb}$ region surrounding the peak location. There were 12 genes in this restricted region, including seven protein-coding, three nonprotein-coding, one microRNA and one small nucleolar RNA gene. Only two of these genes have been observed to have peak expression during the larval stage: dachshund and snoRNA:Me28S-C1237. In the 14\% alcohol environment, the additional QTL encompassed a total of 12 genes, all but one that codes for proteins. Only four of the proteincoding genes are known to have high expression during larval stages. Among these four, only one has any annotated function: CG8223 encodes for a protein that can bind to histones, and is involved in nucleosome assembly. The different QTLs mapped in the 0 and $14 \%$ alcohol

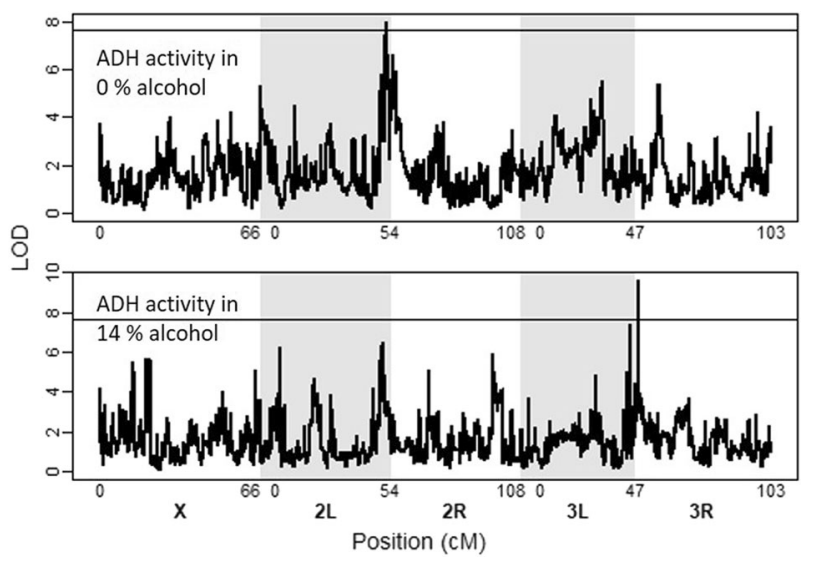

Fig. 2 QTL identified for ADH (alcohol dehydrogenase) activity in larval Drosophila melanogaster developing in substrate with $0 \%$ and $14 \%$ alcohol (top and bottom panels, respectively) after controlling for allelic variation in Adh and delta-1 loci. Black line represents the 95\% LOD score thresholds using 1000 permutation tests.

environments suggested that their effects depended upon what alcohol environments larvae experienced. This pattern is further supported by the intermediate correlations among ADH activity in the three environments (Table 3).

The RILs showed substantial plasticity in ADH activity (Fig. 3). The majority of lines had increased ADH activity when larvae were exposed to higher alcohol concentrations in the larval substrate. There were several lines, however, that exhibited decreased $\mathrm{ADH}$ activity in response to increasing alcohol (Fig. 3). In contrast to ADH activity, we did not detect QTLs for any of the three plasticity measures (Table 1). This was also true when controlling for the allelic effects at the Adh and delta-1 loci. There were several peaks that were close to the global significance threshold, but none that crossed the significance threshold. Interestingly, 
Table 3 Pearson's correlation coefficients between alcohol dehydrogenase $(\mathrm{ADH})$ activity and its plasticity across differing concentrations of alcohol $(0,7$, and $14 \%$ ) in the feeding substrate of larval $D$. melanogaster.

\begin{tabular}{lcclccc}
\hline ADH traits & ADH 0\% & ADH 7\% & ADH 14\% & p07 & p014 & p714 \\
\hline Activity 0\% & 1 & - & - & - & - & - \\
Activity 7\% & $0.57^{*}$ & 1 & - & - & - & - \\
Activity 14\% & $0.47^{*}$ & $0.46^{*}$ & 1 & - & - & - \\
p07 & $-0.44^{*}$ & $0.48^{*}$ & 0.011 & 1 & - & - \\
p014 & $-0.41^{*}$ & -0.027 & $0.61^{*}$ & $0.41^{*}$ & 1 & - \\
p714 & -0.038 & $-0.45^{*}$ & $0.58^{*}$ & $-0.44^{*}$ & $0.64^{*}$ & 1 \\
\hline
\end{tabular}

Plasticity is indicated by p07, p014, and p714, which indicates environments across which plasticity was estimated.

Significant coefficients are marked by $*(p<0.05)$.

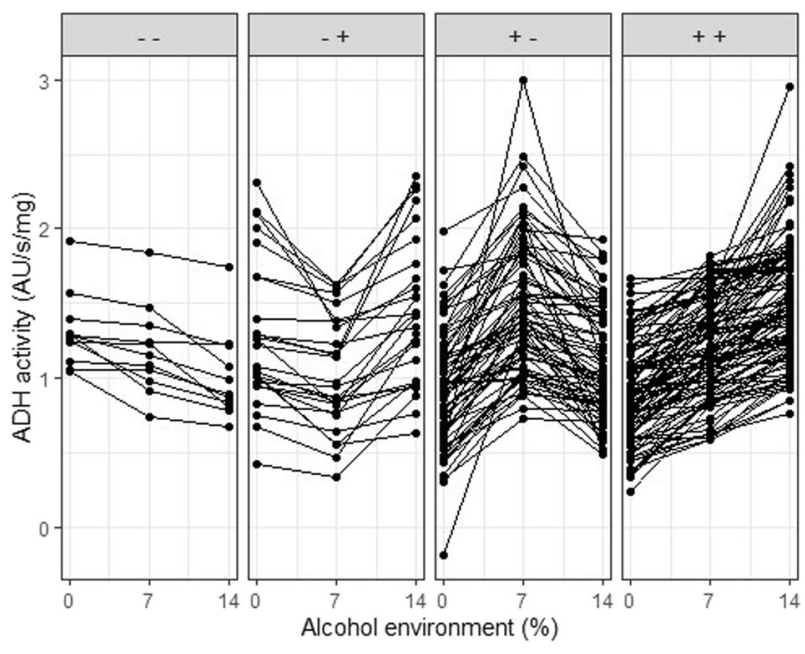

Fig. 3 Reaction norms for ADH (alcohol dehydrogenase) activity in Drosophila melanogaster larvae developing in different concentrations of alcohol in the larval substrate. Each line represents a RIL from the pB2 subpopulation from the Drosophila Synthetic Population Resources. Symbols in each panel represent the sign of ADH plasticity for the $0-7$ and $7-14 \%$ alcohol ranges.

the QTL containing the Adh and delta-1 loci did not contribute to plasticity. Given that some RILs had contrasting patterns of plasticity, we redid the QTL analysis with just the lines that exhibited increasing ADH activity with increasing alcohol separately for each plasticity measure. If different loci were important for increasing or decreasing plasticity, we felt that this might have influenced the significance threshold for any one QTL locus across all RILs. No QTLs were identified for this subset of RILs either.

None of the correlations between ADH activity and plasticity measures were very high (Table 3 ), supporting the pattern that $\mathrm{ADH}$ activity and plasticity have somewhat separate genetic basis. The correlations between the three plasticity measures were also intermediate, suggesting somewhat independent genetic architecture for different plasticity measures. Notably, the correlation between p07 and p714 was negative, capturing the substantial nonlinearity in plastic responses to the range of historical-tonovel alcohol environments.

\section{Discussion}

Using QTL analysis, we investigated the genetic architecture of a functional trait, ADH activity, and its plasticity across historical and novel alcohol environments in Drosophila melanogaster. Our goal was to determine if genetic architecture is the same across environments, and whether plasticity is controlled by environmentally sensitive loci that code for a functional trait, other loci or a combination of both. Across all three environments, ADH activity in fly larvae was largely controlled by a major QTL encompassing the Adh-coding gene and its regulatory loci, delta-l (Table 1 and Fig. 1). The effects of Adh and delta- 1 have been well characterized on ADH activity in flies, and the results from this study demonstrate that their importance is not attenuated by differences in alcohol environments. The positive correlations observed between ADH activity measured in the three environments also supported that the genetic basis for ADH activity is strongly consistent across environments (Table 3). The sequencing coverage information for the founder lines and existing knowledge in the allelic variation in Adh allowed us to test whether the allelic variation at $A d h$ and delta-1 loci was the causal factor in the single QTL (King et al. 2012). There are two major alleles for $A d h$, fast and slow, producing ADH proteins that are different in amino acid sequence and specific activity (Kreitman 1983). Even though the amino acid change does not cause changes in protein quantity, the fast allele is in linkage disequilibrium with other loci in and around the Adh gene that leads to higher protein quantities, including a sequencelength polymorphism in the first intron, delta-1 (Corbin and Maniatis 1990; Laurie and Stam 1994). Because the fast and slow alleles are correlated with delta- 1 in our mapping population, we controlled for their effects simultaneously. After controlling for this allelic variation, the same QTL region showed little correlation with $\mathrm{ADH}$ activity in all three alcohol environments, suggesting that variation in the Adh-coding gene is the most important cause of differences in $\mathrm{ADH}$ activity, regardless of the alcohol environments. 
Controlling for allelic variation in $A d h$ also allowed us to map two additional QTLs for ADH activity, one in the $0 \%$ and the other in $14 \%$ alcohol environments (Table 2 and Fig. 2). In the 0\% alcohol environment, the new QTL overlapped with a QTL previously discovered in adults raised without alcohol exposure, suggesting that its effects were independent of the developmental stages of the fly (King et al. 2012). There were 12 annotated genes in this region, but the molecular function is known for only a few of them. For example, the dachshund gene has broad regulatory roles in eye, leg, and brain development (Martini et al. 2000). In the $14 \%$ alcohol environment, a different QTL was found on the $3 \mathrm{R}$ chromosome. In this region, few of the genes annotated have known functions related to alcohol metabolism. The third chromosome has previously been shown to affect alcohol tolerance. Fry (2014) compared the third chromosome from a temperate population with that from a tropical population after crossing either chromosomes into a common African genetic background, and adults with the temperate third chromosome had higher survival under alcoholic conditions than flies with the tropical third chromosome. There was no additional QTL discovered in the $7 \%$ environment, suggesting that different or the same gene had different effects in the different alcohol environment. Together, the different results from the different alcohol environments indicated that different genetic elements partly control trait variation within historical and across historical and novel environments. Thus, evolution in novel environments may not be entirely constrained by past evolution in historical environments. This is consistent with a large body of evidence from other systems, supporting the importance of cryptic genetic variation in evolution in novel environments (Dlugosch et al. 2015; Hamilton et al. 2015; Paaby and Rockman 2014).

Even though the QTL containing Adh and delta-1, and the two additional ones found in $0 \%$ and the $14 \%$ alcohol environments, explained considerable variation in $\mathrm{ADH}$ activity (34-49\%), none of them showed any significant association with ADH plasticity. The RILs had strong plastic responses to alcohol concentrations in the larvalfeeding substrates (Fig. 3), but we found no QTLs of major effect that explained variation in plastic responses (Table 1). Our ability to map some QTLs in ADH activity suggests that we had enough sample sizes to discover QTLs with large effects, and this suggests that plasticity in $\mathrm{ADH}$ activity was not controlled by a few genes with large effects. The lack of plasticity of QTLs does not, however, suggest that plasticity is under less genetic control than functional trait values, because plasticity could be controlled by many QTLs with small effects. We did not, however, have enough power to detect QTLs with small effects, and it remains unclear whether plasticity in $\mathrm{ADH}$ activity is controlled by a similar set of genes in historical versus novel environments.

The contrasting results for ADH activity and its plasticity suggest that somewhat different genes control for ADH activity in different environments and for plasticity. Similarly, Zhou et al. (2012) found little overlap of genetically based and phenotypically plastic transcript expressions in D. melanogaster, suggesting a different genetic basis for plasticity. This is consistent with other studies investigating genetic structure of plasticity in other systems across historical environments. For example, trait values and plasticity were found to associate with largely different sets of genetic markers for growth and phenology traits in willows (Berlin et al. 2017; Hallingback et al. 2019), for morphology, phenology and reproductive traits in maize (Kusmec et al. 2017) and for growth traits in yeast (Yadav et al. 2016). Even though QTLs for trait values and plasticity can differ greatly in some organisms, it is not uncommon to find plasticity genes to co-locate with trait QTLs, such was found for biomass in hybrid willows (Berlin et al. 2017) and for phenology in maize (Li et al. 2016). These examples and our data highlight that plasticity can evolve independently from trait values in some environments, and this pattern is robust across both historical and novel environments.

In conclusion, we found that the genetic basis of $\mathrm{ADH}$ activity was largely determined by a QTL containing the gene $A d h$ and its regulatory locus, delta-1. This QTL is important in ADH activity across both historical and novel concentrations of alcohol in larval substrates. We also found that the Adh-coding gene is the most important factor influencing variation in $\mathrm{ADH}$ activity, but different minor QTLs were important in different alcohol environments. These patterns indicate that $\mathrm{ADH}$ activity could evolve unconstrained by trait evolution in either historical or novel environments. Although fly RILs expressed plastic responses to alcohol concentrations, plasticity is likely controlled by many loci with medium-to-small effects, and is largely controlled by separate genetic elements than those controlling ADH activity. Studies in additional systems are needed to determine if this is a common pattern for functional traits and their plasticity. As demonstrated in this study, established mapping resources could be used for detailed genetic dissection of functional traits and plasticity.

\section{Data availability}

Data are available from the Dryad Digital Repository https://doi.org/10.5061/dryad.cnp5hqc2k.

Acknowledgements The authors thank J. Belote, S. Dorus, and S. Pitnick for providing facilities to rear the flies. J. Friedman, S. Pitnick, and K. Segraves also provided valuable discussions throughout the course of the study. We thank three anonymous reviewers for their 
insightful comments that greatly help refine the paper. Funding for the research was provided by NSF 1556568 and 1655544 to DMA.

\section{Compliance with ethical standards}

Conflict of interest The authors declare that they have no conflict of interest.

Publisher's note Springer Nature remains neutral with regard to jurisdictional claims in published maps and institutional affiliations.

\section{References}

Badyaev AV (2005) Stress-induced variation in evolution: from behavioural plasticity to genetic assimilation. Proc Biol Sci 272 (1566): 877-886.

Berlin S, Hallingback HR, Beyer F, Nordh NE, Weih M, RonnbergWastljung AC (2017) Genetics of phenotypic plasticity and biomass traits in hybrid willows across contrasting environments and years. Ann Bot 120(1):87-100

Bhatia A, Yadav A, Zhu C, Gagneur J, Radhakrishnan A, Steinmetz LM et al. (2014) Yeast growth plasticity is regulated by environment-specific multi-QTL interactions. G3 (Bethesda) 4 (5):769-777

Blows MW (2007) A tale of two matrices: multivariate approaches in evolutionary biology. J Evolut Biol 20(1):1-8

Callahan HS, Waller DM (2000) Phenotypic integration and the plasticity of integration in an amphicarpic annual. Int J plant Sci 161(1):89-98

Chevin L-M, Hoffmann AA (2017) Evolution of phenotypic plasticity in extreme environments. Philos Trans R Soc B Biol Sci 372 (1723):20160138

Chevin LM (2013) Genetic constraints on adaptation to a changing environment. Evolution 67(3):708-721

Corbin V, Maniatis T (1990) Identification of cis-regulatory elements required for larval expression of the Drosophila melanogaster alcohol dehydrogenase gene. Genetics 124(3):637-646

DeWitt TJ, Scheiner SM (eds) (2004) Phenotypic plasticity: functional and conceptual approaches. Oxford University Press, New York

Dlugosch KM, Anderson SR, Braasch J, Cang FA, Gillette HD (2015) The devil is in the details: genetic variation in introduced populations and its contributions to invasion. Mol Ecol 24 (9):2095-2111

Fischer EK, Ghalambor CK, Hoke KL (2016) Plasticity and evolution in correlated suites of traits. J Evol Biol 29(5):991-1002

Freriksen A, Seykens D, Scharloo W, Heinstra P (1991) Alcohol dehydrogenase controls the flux from ethanol into lipids in Drosophila larvae. A 13C NMR study. J Biol Chem 266 (32):21399-21403

Fry JD (2014) Mechanisms of naturally evolved ethanol resistance in Drosophila melanogaster. J Exp Biol 217(Pt 22):3996-4003

Geer BW, Heinstra PW, McKechnie SW (1993) The biological basis of ethanol tolerance in Drosophila. Comp Biochem Physiol Part B Comp Biochem 105(2):203-229

Geer BW, Langevin ML, McKechnie SW (1985) Dietary ethanol and lipid synthesis in Drosophila melanogaster. Biochem Genet 23 (7-8):607-622

Gibson JB, May TW, Wilks AV (1981) Genetic variation at the alcohol dehydrogenase locus in Drosophila melanogaster in relation to environmental variation: ethanol levels in breeding sites and allozyme frequencies. Oecologia 51(2):191-198
Gomulkiewicz R, Holt RD, Barfield M, Nuismer SL (2010) Genetics, adaptation, and invasion in harsh environments. Evol Appl 3 (2):97-108

Hallingback HR, Berlin S, Nordh NE, Weih M, Ronnberg-Wastljung AC (2019) Genome wide associations of growth, phenology, and plasticity traits in willow [Salix viminalis (L.)]. Front Plant Sci 10:753

Hamilton JA, Okada M, Korves T, Schmitt J (2015) The role of climate adaptation in colonization success in Arabidopsis thaliana. Mol Ecol 24(9):2253-2263

Hangartner S, Hoffmann AA (2016) Evolutionary potential of multiple measures of upper thermal tolerance in Drosophila melanogaster. Funct Ecol 30(3):442-452

Heinstra PW, Seykens D, Freriksen A, Geer BW (1990) Metabolic physiology of alcohol degradation and adaptation in Drosophila larvae as studied by means of carbon-13 nuclear magnetic resonance spectroscopy. Insect. Biochemistry 20(4):343-348

Husby A, Visser ME, Kruuk LE (2011) Speeding up microevolution: the effects of increasing temperature on selection and genetic variance in a wild bird population. PLoS Biol 9(2):e1000585

Jain K, Stephan W (2017) Rapid adaptation of a polygenic trait after a sudden environmental shift. Genetics 206(1):389-406

Kapoun AM, Geer BW, Heinstra PW, Corbin V, McKechnie SW (1990) Molecular control of the induction of alcohol dehydrogenase by ethanol in Drosophila melanogaster larvae. Genetics 124(4):881-888

King EG, Merkes CM, McNeil CL, Hoofer SR, Sen S, Broman KW et al. (2012) Genetic dissection of a model complex trait using the Drosophila Synthetic population resource. Genome Res 22 (8):1558-1566

Kopp M, Matuszewski S (2014) Rapid evolution of quantitative traits: theoretical perspectives. Evolut Appl 7(1):169-191

Kreitman M (1983) Nucleotide polymorphism at the alcoholdehydrogenase locus of Drosophila melanogaster. Nature 304 (5925):412-417

Kusmec A, Srinivasan S, Nettleton D, Schnable PS (2017) Distinct genetic architectures for phenotype means and plasticities in Zea mays. Nat Plants 3(9):715-723

Lacaze X, Hayes PM, Korol A (2009) Genetics of phenotypic plasticity: QTL analysis in barley, Hordeum vulgare. Heredity (Edinb) 102(2):163-173

Lande R (2009) Adaptation to an extraordinary environment by evolution of phenotypic plasticity and genetic assimilation. J Evol Biol 22(7):1435-1446

Laurie CC, Stam LF (1994) The effect of an intronic polymorphism on alcohol dehydrogenase expression in Drosophila melanogaster. Genetics 138(2):379-385

Levis NA, Isdaner AJ, Pfennig DW (2018) Morphological novelty emerges from pre-existing phenotypic plasticity. Nat Ecol Evol 2 (8): 1289-1297

Levis NA, Pfennig DW (2016) Evaluating 'plasticity-first'evolution in nature: key criteria and empirical approaches. Trends Ecol Evol 31(7):563-574

Levis NA, Pfennig DW (2019) Plasticity-led evolution: evaluating the key prediction of frequency-dependent adaptation. Proc R Soc B 286(1897):20182754

Li YX, Li C, Bradbury PJ, Liu X, Lu F, Romay CM et al. (2016) Identification of genetic variants associated with maize flowering time using an extremely large multi-genetic background population. Plant J 86(5):391-402

Malherbe Y, Kamping A, van Delden W, van de Zande L (2005) ADH enzyme activity and Adh gene expression in Drosophila melanogaster lines differentially selected for increased alcohol tolerance. J Evol Biol 18(4):811-819 
Markow TA (2015) The secret lives of Drosophila flies. Elife 4:1-9

Martini SR, Roman G, Meuser S, Mardon G, Davis RL (2000) The retinal determination gene, dachshund, is required for mushroom body cell differentiation. Development 127(12):2663-2672

McCairns RJ, Bernatchez L (2010) Adaptive divergence between freshwater and marine sticklebacks: insights into the role of phenotypic plasticity from an integrated analysis of candidate gene expression. Evolution 64(4):1029-1047

McDonald JF, Anderson SM, Santos M (1980) Biochemical differences between products of the Adh locus in Drosophila. Genetics 95(4):1013-1022

Mckechnie SW, Geer BW (1984) Regulation of alcohol dehydrogenase in Drosophila melanogaster by dietary alcohol and carbohydrate. Insect Biochem 14(2):231-\&

Mercot H, Defaye D, Capy P, Pla E, David JR (1994) Alcohol tolerance, ADH activity, and ecological niche of Drosophila species. Evolution 48(3):746-757

Merila J, Hendry AP (2014) Climate change, adaptation, and phenotypic plasticity: the problem and the evidence. Evol Appl 7(1):1-14

Nussey DH, Postma E, Gienapp P, Visser ME (2005) Selection on heritable phenotypic plasticity in a wild bird population. Science 310(5746):304-306

Paaby AB, Rockman MV (2014) Cryptic genetic variation: evolution's hidden substrate. Nat Rev Genet 15(4):247-258

Pigliucci M (2001) Phenotypic plasticity: beyond nature and nurture. The John Hopkins University Press, Baltimore

R Core Team (2017) R: a language and environment for statistical computing. R Foundation for Statistical Computing, Vienna, Austria, https://www.R-project.org/

Schaum CE, Collins S (2014) Plasticity predicts evolution in a marine alga. Proc Biol Sci 281(1793):20141486

Scheiner SM (1993) Genetics and evolution of phenotypic plasticity. Annu Rev Ecol Syst 24:35-68

Schlichting CD, Pigliucci M (1998) Phenotypic evolution: a reaction norm perspective. Sinauer Associates Incorporated, Sunderland, Massachusetts

Schluter D (1996) Adaptive radiation along genetic lines of least resistance. Evolution 50(5):1766-1774

Siddiq MA, Thornton JW (2019) Fitness effects but no temperaturemediated balancing selection at the polymorphic $A D H$ gene of
Drosophila melanogaster. Proc Natl Acad Sci USA 116 (43):21634-21640

Siddiq MA, Loehlin DW, Montooth KL, Thornton JW (2017) Experimental test and refutation of a classic case of molecular adaptation in Drosophila melanogaster. Nat Ecol Evol 1:25. (2017)

Snell-Rood EC, Van Dyken JD, Cruickshank T, Wade MJ, Moczek AP (2010) Toward a population genetic framework of developmental evolution: the costs, limits, and consequences of phenotypic plasticity. Bioessays 32(1):71-81

Thompson JN, Kaiser TN (1977) Selection acting upon slowmigrating $\mathrm{ADH}$ alleles differing in enzyme activity. Heredity 38(2):191-195

Thurmond J, Goodman JL, Strelets VB, Attrill H, Gramates LS, Marygold SJ, Matthews BB, Millburn G, Antonazzo G, Trovisco V, Kaufman TC, Calvi BR, the FlyBase Consortium (2019) FlyBase 2.0: the next generation. Nucleic Acids Res 47(D1): D759-D765

Tonsor SJ, Scheiner SM (2007) Plastic trait integration across a $\mathrm{CO}_{2}$ gradient in Arabidopsis thaliana. Am Nat 169(5):E119-140

Via S, Gomulkiewicz R, De Jong G, Scheiner SM, Schlichting CD, Van Tienderen PH (1995) Adaptive phenotypic plasticity: consensus and controversy. Trends Ecol Evolution 10(5):212-217

Wang SP, Althoff DM (2019) Phenotypic plasticity facilitates initial colonization of a novel environment. Evolution 73(2):303-316

Wei X, Zhang J (2017) The genomic architecture of interactions between natural genetic polymorphisms and environments in yeast growth. Genetics 205(2):925-937

West-Eberhard MJ (2003) Developmental plasticity and evolution. Oxford University Press, New York

Yadav A, Dhole K, Sinha H (2016) Genetic regulation of phenotypic plasticity and canalisation in yeast growth. PLoS ONE 11(9): e0162326

Yeh PJ, Price TD (2004) Adaptive phenotypic plasticity and the successful colonization of a novel environment. Am Nat 164 (4):531-542

Zhou S, Campbell TG, Stone EA, Mackay TF, Anholt RR (2012) Phenotypic plasticity of the Drosophila transcriptome PLoS Genet 8:e1002593 